\title{
Formação para nutricionistas que atuam no Programa Nacional de Alimentação Escolar: uma avaliação da efetividade
}

\author{
Training for nutritionists working on the Brazilian School \\ Nutrition Program: an evaluation of its effectiveness
}

Ana Luiza Sander Scarparo ${ }^{1}$

Viviani Ruffo de Oliveira ${ }^{2}$

Jaqueline Marcela Villafuerte Bittencourt ${ }^{3}$

Eliziane Nicolodi Francescato Ruiz ${ }^{1}$

Patrícia Fogaça Fernandes ${ }^{1}$

Júlia Zanchetta Zys ${ }^{1}$

Cileide Cunha Moulin ${ }^{2}$

${ }^{1}$ Centro Colaborador em Alimentação e Nutrição do Escolar, Universidade Federal do Rio Grande do Sul. Rua Ramiro Barcelos 2400/4 ${ }^{\circ}$, Santana. 90035-003 Porto Alegre RS. capacitacecane@ufrgs.br ${ }^{2}$ Departamento de Medicina Interna, Faculdade de Medicina, Universidade Federal do Rio Grande do Sul.

3 Programa de PósGraduação em Educação, Faculdade de Educação, Universidade Federal do Rio Grande do Sul.

\begin{abstract}
This article seeks to verify the effectiveness of training for nutritionists conducted by the Cooperation Center for Student Food and Nutrition of the Federal University of Rio Grande do Sul (CECANES UFRGS), between 2007 and 2009. The focus of the evaluation was to observe the perception of those subjects with regard to achieving the objectives of the training and the potential of the training topics covered in the training in order to change the day-to-day work of the Brazilian School Nutrition Program (PNEA). Methodology developed by the CECANES UFRGS team was used for the evaluation of effectiveness. Data collection was conducted with 118 nutritionists who work in school nutrition in the state of Rio Grande do Sul by filling out an electronic questionnaire specifically designed for this study. In the analysis of the results it was revealed that the training fulfilled its objectives $(P<0.05)$, making the assignments and what is expected of the professionals at PNAE clear to more than $95 \%$ of the nutritionists. When the participants were asked about the obstacles encountered, they reported difficulty in adapting the theory covered in the training with the reality in which they act. The themes addressed contributed to the promotion of changes in the implementation of management practices in the PNAE.
\end{abstract}

Key words Nutritionist, Training, Effectiveness, School nutrition
Resumo O objetivo deste artigo é verificar a efetividade das formações de nutricionistas realizadas pelo Centro Colaborador em Alimentação e Nutrição do Escolar da Universidade Federal do Rio Grande do Sul (CECANES UFRGS), entre 2007 e 2009. O foco da avaliação foi a percepção destes atores quanto à consecução dos objetivos da formação e quanto ao potencial das temáticas da formação em alterar o dia a dia de trabalho no Programa Nacional de Alimentação Escolar (PNAE). Para avaliação da efetividade foi utilizada a metodologia elaborada pela equipe do CECANES da UFRGS. A coleta de dados foi realizada com 118 nutricionistas, que atuam na alimentação escolar no RS, através de preenchimento de questionário eletrônico elaborado especificamente para esse trabalho. Observou-se que a formação cumpriu seus objetivos $(P<0,05)$, deixando claro para mais de 95\% dos nutricionistas as atribuições e o que se espera do profissional no PNAE. Quando questionados sobre os obstáculos encontrados, os participantes relatam dificuldade em adaptar a teoria abordada na formação à realidade em que atuam. As temáticas trabalhadas contribuíram para a promoção de mudanças na execução do PNAE, principalmente em termos de difusão e atualização das normas e objetivos e na troca de experiências e práticas eficientes de gestão.

Palavras-chave Nutricionista, Formação, Efetividade, Alimentação escolar 


\section{Introdução}

Para atingir as metas do milênio para Educação e Saúde, e qualificar a gestão e o controle social do Programa Nacional de Alimentação Escolar (PNAE), o Fundo Nacional de Desenvolvimento da Educação (FNDE), baseado na Portaria Interministerial 1.010 de 2006, firmou parcerias com Instituições Federais de Ensino Superior para a criação dos Centros Colaboradores em Alimentação e Nutrição do Escolar (CECANES), a fim de auxiliar na implantação das diretrizes do PNAE e na consolidação da Política Nacional de Segurança Alimentar e Nutricional (PNSAN) no ambiente escolar ${ }^{1,2}$.

Os CECANES vêm, desde então, prestando apoio técnico e operacional na implementação da alimentação saudável nas escolas, agindo através da assessoria e do acompanhamento da execução do Programa nos municípios, de pesquisas na área de alimentação escolar (AE) e da formação de profissionais envolvidos no PNAE ${ }^{1,2}$.

Com relação à formação, parte-se do entendimento de que ao gerar envolvimento, reflexão, postura crítica e ética sobre as atividades desenvolvidas pelos participantes em suas realidades, este é um processo que tem a possibilidade de qualificar a execução das políticas públicas e potencializar o alcance do cumprimento de suas diretrizes e princípios ${ }^{2}$. Nesse sentido, foram desenvolvidas ações visando aprimorar, de forma contextualizada, os conhecimentos e as habilidades básicas dos diferentes atores envolvidos no PNAE. Dentre os atores, cada vez mais emerge e destaca-se no Programa a atuação do nutricionista que, com seu papel de responsável técnico pela $\mathrm{AE}^{3}$, tem a possibilidade de atuar como peça chave para a promoção da Segurança Alimentar e Nutricional (SAN) no ambiente da escola.

Vale também destacar, como parte importante do processo de formação, a etapa de avaliação, a qual constitui-se um procedimento de produção de informações sobre os resultados da ação, e uma forma de verificar a sua coerência com os objetivos previstos. Esta é uma estratégia importante da gestão pública, pois através dela pode-se informar aos dirigentes, legisladores e público alvo a efetividade e a relevância dos programas públicos ${ }^{4}$.

Draibe $^{5}$ sugere a existência de quatro tipos de indicadores passíveis de serem utilizados na avaliação, sendo eles: indicadores de desempenho, eficiência, eficácia e efetividade. A efetividade é utilizada para expressar o grau de consecução dos objetivos específicos de um projeto, ou seja, em que medida os recursos, atividades e produtos conseguiram os efeitos diretos almejados, em um determinado período de tempo, independentemente dos custos implicados. Ao avaliar a efetividade verifica-se, também, a percepção dos resultados expressos pelos sujeitos que receberam as ações. A efetividade é considerada uma medida ampla dos efeitos da política, programa ou ação realizada ${ }^{6}$.

Tendo em vista a escassez de estudos com enfoque na formação de atores do PNAE e a necessidade de avaliar as formações do CECANES UFRGS, desenvolveu-se este trabalho com o objetivo de verificar a percepção destes atores quanto à consecução dos objetivos da formação e quanto ao potencial das temáticas abordadas na formação em alterar o cotidiano de trabalho no PNAE. Além disso, procurou-se identificar as possíveis dificuldades encontradas no desempenho de suas atribuições junto a esta política pública.

\section{Métodos}

O CECANES UFRGS, entre 2007 a 2009, formou, dentro de um processo de educação que é continuada e que não se encerra no âmbito da graduação, um total de 362 nutricionistas que atuam no PNAE no RS, em 10 turmas. As formações seguiram a metodologia planejada, em 2007, pelos CECANES e FNDE, e sistematizada pelo CECANES da Universidade Federal de São Paulo $(\mathrm{UNIFESP})^{7}$, contendo atividades teóricas e práticas, entre elas: exposições dialogadas, dinâmicas de grupos, estudos de casos e reflexões individuais. Os três dias, totalizando 24 horas de formação, priorizaram a participação, a troca de experiência e a reflexão crítica sobre as atribuições, valorizando os conhecimentos dos participantes. Os temas abordados foram: Direito $\mathrm{Hu}-$ mano à Alimentação Adequada, SAN, histórico e panorama atual do PNAE, as atribuições específicas do nutricionista e seu trabalho na AE.

Para a avaliação da efetividade das formações, foi desenvolvida uma proposta metodológica, de 2009 a 2010, por uma equipe constituída por nutricionistas e técnicos da área de avaliação de políticas públicas e da pedagogia. Cabe destacar que este trabalho é oriundo do produto do CECANES UFRGS intitulado "Metodologia para avaliação da efetividade das capacitações dos agentes do PNAE", sendo fomentado pelo FNDE.

As dimensões analíticas da pesquisa foram desenhadas a partir da legislação vigente do 
$\mathrm{PNAE}^{3,8}$, da metodologia elaborada pelo CECANES UNIFESP ${ }^{7}$ e dos materiais de apoio ao curso. Esta lógica seguiu a premissa de que não se fazem avaliações longe dos objetivos e princípios norteadores de cada objeto de pesquisa, sob pena de construir uma avaliação distorcida ou preconceituosa ${ }^{9}$. O Quadro 1 apresenta a sistematização das dimensões analíticas e suas categorias.

O instrumento, elaborado e utilizado para a coleta de dados, teve como escopo as dimensões analíticas e apresentou dezenove questões, subdivididas nos seguintes eixos: perfil, formação anterior, trabalho no PNAE, formação e percepção final.

A coleta de dados foi realizada em 2010. Foi enviada uma correspondência eletrônica para os nutricionistas formados, entre 2007 e 2009, apresentando a pesquisa, seus objetivos, e solicitando a utilização de um link (www.encuestafacil.com) ${ }^{10}$ para acessar uma ferramenta e responder o questionário. Não foi necessária a identificação do participante e foi ressaltada a relevância da participação para o sucesso da avaliação da efetividade das formações. Para Freitas et al. ${ }^{11}$ utilizando a internet o pesquisador não está mais limitado pelas restrições de tempo, custo e distância, possuindo um acesso mundial praticamente instantâneo, com despesas mínimas.

Para a análise estatística dos dados foi utilizado o software SPSS (Statistical Package for the Social Sciences) versão 17.0. As variáveis foram descritas através de frequências absolutas e relativas. Para as comparações, foi aplicado o teste qui-quadrado, sendo o nível de significância estatística considerado de $5 \%(\mathrm{p}<0,05)$.

\section{Resultados e discussão}

Dos 362 contatos eletrônicos 126 (34,7\%) responderam o questionário. Entretanto, oito $(6,3 \%)$ foram desconsiderados, pois estavam incompletos. Assim, foram considerados os resultados de 118 questionários preenchidos completamente pelos participantes. Com relação ao perfil dos participantes, destaca-se que a maioria era do sexo feminino, com idade entre 20 e 30 anos (47\%), 74,6\% eram responsáveis em apenas um município, mediana de 4 anos de atuação e, em média, 24,8 $( \pm 11,6)$ horas semanais, e $8,5 \%$ não atuavam mais na $\mathrm{AE}$.

O percentual de retorno deste trabalho, $34,8 \%$, está acima da média para este tipo de instrumento, validando o uso desta metodologia de pesquisa ${ }^{12}$. Segundo Galan e Vernette citado por Freitas et al. ${ }^{11}$, a taxa de retorno padrão de um e-mail é comparável àquelas obtidas via modo postal, sendo de 7 a $13 \%$ sobre o total enviado, podendo aumentar de acordo com a população questionada.

Ressalta-se que em 2008, o CECANES UFRGS utilizou o meio eletrônico para realizar a coleta de dados da pesquisa que verificou o perfil dos profissionais nutricionistas que atuavam no PNAE no Estado. O instrumento foi enviado para 387 nutricionistas e ao final obteve-se o retorno

Quadro 1. Dimensões analíticas para a avaliação da efetividade da Formação para nutricionistas que atuam no Programa Nacional de Alimentação Escolar. Porto Alegre - RS, 2010.

\begin{tabular}{|c|l|}
\hline \multicolumn{1}{|c|}{ Dimensões Analíticas } & \multicolumn{1}{|c|}{ Categorias analíticas } \\
\hline $\begin{array}{c}1^{\text {a }} \text { dimensão } \\
\text { Dos objetivos do PNAE } \\
\text { Dos objetivos das formações }\end{array}$ & $\begin{array}{l}\text { Atribuições do Nutricionista } \\
\text { Articulação do PNAE e Política de SAN }\end{array}$ \\
\hline $\begin{array}{c}2^{\text {a }} \text { dimensão } \\
\text { Da gestão do Programa }\end{array}$ & $\begin{array}{l}\text { Funcionamento } \\
\text { Execução das atividades } \\
\text { Licitações } \\
\text { Prestação de contas } \\
\text { Entrosamento com outros atores } \\
\text { Controle social } \\
\text { Valorização profissional }\end{array}$ \\
\hline $3^{\text {a dimensão }}$ Dos conhecimentos técnicos & $\begin{array}{l}\text { Gestão de conflitos cotidianos } \\
\text { Atualização técnica } \\
\text { Elaboração de cardápios } \\
\text { Teste de Aceitabilidade } \\
\text { Avaliação Nutricional }\end{array}$ \\
& \\
\hline
\end{tabular}


de 171 profissionais (44,19\%). Além disso, uma sondagem nas formações de 2008 e 2009 encontrou que a maioria tinha acesso à internet diária ou semanalmente, respectivamente, $94,1 \%$ e $96,5 \%$ dos participantes.

\section{A formação como possibilidade para ampliar a atuação do nutricionista junto ao PNAE}

De acordo com os resultados apresentados na Tabela 1, observa-se que, de modo geral, a formação cumpriu seus objetivos, demonstrando diferença estatística significativa $(\mathrm{P}<0,05)$ em todos os itens avaliados. A partir dessa informação pode-se sugerir que a formação foi efetiva, pois se verificou adequada satisfação e apreensão de conhecimento pelos participantes diante de cada um dos objetivos do curso descritos.

Quando indagado aos nutricionistas sobre inovações específicas que a formação possibilitou em relação às suas atividades, conforme se pode observar na Figura 1, aproximadamente $60 \%$ dos respondentes referiram informações relacionadas aos procedimentos de compra dos produtos e também de elaboração de cardápios para a AE. Esta informação pode ser analisada sob o prisma de que essas duas temáticas não são abordadas satisfatoriamente ou de forma contextualizada no currículo acadêmico de muitos cursos de nutrição, sugerindo uma carência específica na formação dos nutricionistas.

Quando questionados se consideravam sua formação acadêmica suficiente para atuação no
PNAE, a grande maioria $(78,8 \%)$ referiu como insuficiente. Na pesquisa do CECANES, realizada por $\operatorname{Voos}^{13}$, em 2009, 95\% dos nutricionistas inferiram a necessidade de uma formação específica para se atuar na AE. Sendo que apenas 14\% referiram que sua formação inicial foi suficiente para o desempenho da função. Este dado é de grande relevância, não necessariamente significando que as Universidades não estejam preparadas para a formação do nutricionista na área de AE, mas sim que se trata de uma política pública que se renova e adapta periodicamente às necessidades de seus usuários. Isto, no entanto, exige que as instituições de ensino abordem o PNAE durante o processo de formação dos acadêmicos não apenas em aulas expositivas, mas também em atividades práticas, como projetos e estágios, que exercitem em ato a práxis e a dinamicidade do processo.

Cabe referir que, em muitos municípios, o nutricionista desempenha a função de forma isolada, sem pares na profissão com quem poderia discutir, dirimir dúvidas e solucionar conflitos. As formações, além de constituírem um espaço importante de troca de informações e experiências, também têm como proposição o estímulo para o trabalho em grupo e a abertura para a intersetorialidade. Neste sentido, observou-se que $46,6 \%$ $(\mathrm{n}=55)$ dos participantes mencionam possibilidades relacionadas ao entrosamento com outros atores da política e 39\% ( $\mathrm{n}=46)$ destaca a melhora na gestão dos conflitos cotidianos.

Políticas públicas, tal como o PNAE, adquirem concretude pelas mãos dos "recursos hu-

Tabela 1. Avaliação da consecução dos objetivos específicos da formação para nutricionistas que atuam no Programa Nacional de Alimentação Escolar. Porto Alegre - RS, 2010.

\begin{tabular}{lccc}
\hline \multicolumn{1}{c}{ Variáveis } & $\begin{array}{c}\text { Concordo } \\
\text { plenamente/ } \\
\text { Concordo } \\
\%(\mathbf{n})\end{array}$ & $\begin{array}{c}\text { Discordo } \\
\text { parcialmente/ } \\
\text { Discordo } \\
\%(\mathbf{n})\end{array}$ & $\mathbf{P}^{*}$ \\
\hline $\begin{array}{l}\text { Dimensão 1 } \\
\begin{array}{l}\text { A capacitação deixou claras as atribuições do Nutricionista } \\
\text { no PNAE. }\end{array}\end{array} \quad 95,9(116)$ & $4,1(5)$ & $<0,001$ \\
$\begin{array}{l}\text { A apresentação do PNAE mostrou uma dimensão ampliada } \\
\text { sobre o que se espera do Nutricionista }\end{array}$ & $96,6(113)$ & $3,4(4)$ & $<0,001$ \\
$\begin{array}{l}\text { A capacitação ampliou seu entendimento sobre a relação } \\
\text { entre o PNAE e Segurança Alimentar e Nutricional (SAN). }\end{array}$ & $88,9(104)$ & $11,1(13)$ & $<0,001$ \\
$\begin{array}{l}\text { A capacitação proporcionou atualização nos seus } \\
\text { conhecimentos em Nutrição. }\end{array}$ & $86,2(100)$ & $13,2(16)$ & $<0,001$ \\
$\begin{array}{l}\text { A capacitação foi fundamental para melhorar o seu } \\
\text { cotidiano de trabalho. }\end{array}$ & $86,2(100)$ & $13,2(16)$ & $<0,001$
\end{tabular}

* Teste qui-quadrado de ajustamento 
Nas questões relacionadas ao entrosamento com os outros atores da política

Na gestão de conflitos cotidianos dimentos relacionados ao processo licitatório

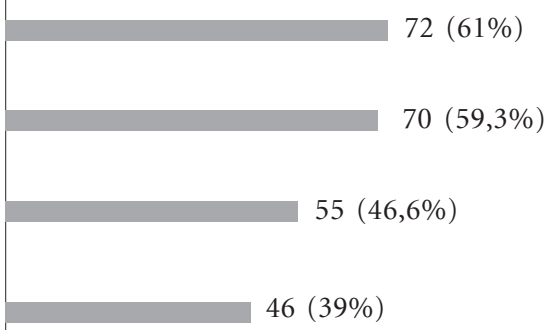

Outro

Não possibilitou novas formas de execução das tarefas
$9(7,6 \%)$

$6(5,1 \%)$

Número de nutricionistas

Figura 1. Distribuição das respostas dos profissionais quanto às inovações possibilitadas pela formação do CECANES. Porto Alegre - RS, 2010.

manos" que as operam no cotidiano do espaço entre o Estado e a sociedade. No entanto, fica claro que na medida em que o Estado assume a responsabilidade social que lhe é devida, também é exigido que os trabalhadores e agentes que a executam, adotem não mais o status de "recursos" e sim de atores (e autores) que assumem compromissos com as necessidades dinâmicas da sociedade ${ }^{14}$. Compromissos estes, que impõem aos profissionais o desafio de adquirir, continuamente, conhecimentos, novas habilidades e atitudes necessárias para o desenvolvimento de suas atividades diárias ${ }^{15}$.

Isto tudo, por sua vez, coloca o processo de formação permanente dessas pessoas como um eixo central para o sucesso das políticas ${ }^{14}$. Nesse sentido, e diante dos resultados aqui apresentados, é possível apreender que os espaços oferecidos pelas formações, quando bem aproveitados, são lócus privilegiados ao possibilitar a problematização de concepções vigentes e a construção de conhecimentos, a reflexão sobre práticas, a proposição de estratégias que articulem atores, setores e políticas envolvidos no Programa e a ampliação da capacidade de enfrentamento das situações cotidianas.

\section{A formação do nutricionista contribuindo para operar mudanças na execução do PNAE}

Questionou-se no instrumento de nutricionistas como, após a formação oferecida pelo CECANES, o profissional classificava sua atua- ção no município, de acordo com as atribuições do nutricionista no PNAE. A Tabela 2 apresenta a avaliação dos profissionais. Este momento do trabalho revela pontos importantes da avaliação de efetividade, considerando-se a capacidade da formação em alterar a realidade.

Através dos resultados pode-se observar que algumas categorias apresentaram diferença estatística significativa $(\mathrm{P}<0,05)$ entre o "sempre desenvolvi" e "desenvolvo de forma mais eficiente", apontando que não houve aprimoramento na forma de execução das atividades. Destaca-se que estes são temas (excetuando-se a Licitação) que não possuíram um maior aprofundamento nas formações. Assim, desde 2010, o CECANES UFRGS vem desenvolvendo cursos de atualização para nutricionistas que participaram de formações, em anos anteriores. Em 2011, o enfoque foi o controle higiênico-sanitário na AE.

Com relação às atividades que não eram desenvolvidas pelos nutricionistas e passaram a ser executadas após a formação, observou-se diferença significativa para a atribuição "Acompanhamento das compras da agricultura familiar". De fato, após a regulamentação para compra de produtos da agricultura familiar, utilizando no mínimo 30\% do recurso financeiro do PNAE, havia muitas dúvidas e resistências que impediam a sua plena implementação. Nesse sentido, a formação possibilitou esclarecimentos sobre a nova legislação e o processo operacional de compra e venda desses produtos ${ }^{3,8}$.

Uma atribuição que merece ser analisada separadamente é a "Aplicação do teste de aceitabi- 
Tabela 2. Atuação do nutricionista no município, de acordo com suas atribuições no PNAE, após a formação. Porto Alegre - RS, 2010.

\begin{tabular}{|c|c|c|c|}
\hline Variáveis & $\begin{array}{c}\text { Sempre } \\
\text { desenvolvi } \\
\%(\mathbf{n})\end{array}$ & $\begin{array}{c}\text { Desenvolvo de } \\
\text { forma mais eficiente } \\
\%(\mathbf{n})\end{array}$ & $\mathbf{p}^{*}$ \\
\hline \multicolumn{4}{|l|}{ Dimensão 2} \\
\hline Coordenação técnica das ações relacionadas com AE. & $33,9(40)$ & $40,7(48)$ & 0,456 \\
\hline Visitas regulares às escolas. & $61,0(72)$ & $24,6(29)$ & $<0,001$ \\
\hline $\begin{array}{l}\text { Acompanhamento das compras dos produtos alimentícios } \\
\text { - Licitação. }\end{array}$ & $50,8(60)$ & $26,3(31)$ & 0,003 \\
\hline Acompanhamento das compras da agricultura familiar. & $24,6(29)$ & $26,3(31)$ & 0,897 \\
\hline Visita técnica aos fornecedores. & $28,0(33)$ & $18,6(22)$ & 0,177 \\
\hline $\begin{array}{l}\text { Desenvolvimento de capacitação de Manipuladores de } \\
\text { Alimentos (Merendeiras). }\end{array}$ & $55,9(66)$ & $28,8(34)$ & 0,002 \\
\hline Interação e assessoramento (trabalho em equipe) ao CAE. & $34,7(41)$ & $36,4(43)$ & 0,913 \\
\hline \multicolumn{4}{|l|}{ Dimensão 3} \\
\hline Planejamento de cardápios, conforme a legislação vigente. & $37,3(44)$ & $45,8(54)$ & 0,363 \\
\hline $\begin{array}{l}\text { Aplicação do teste de aceitabilidade seguindo critérios da } \\
\text { legislação. }\end{array}$ & $11,9(14)$ & $19,5(23)$ & 0,188 \\
\hline Elaboração de Manual de Boas Práticas. & $16,1(19)$ & $16,9(20)$ & 1,000 \\
\hline $\begin{array}{l}\text { Verificação do perfil da população atendida, identificando } \\
\text { crianças com necessidades nutricionais especiais. }\end{array}$ & $19,5(23)$ & $24,6(29)$ & 0,488 \\
\hline Monitoramento do estado nutricional dos estudantes. & $25,4(30)$ & $22,9(27)$ & 0,791 \\
\hline Variáveis & $\begin{array}{c}\text { Passei a } \\
\text { desenvolver } \\
\%(\mathbf{n})\end{array}$ & $\begin{array}{c}\text { Ainda não } \\
\text { desenvolvo } \\
\%(\mathbf{n})\end{array}$ & $\mathbf{p}^{*}$ \\
\hline \multicolumn{4}{|l|}{ Dimensão 2} \\
\hline Coordenação técnica das ações relacionadas com AE. & $10,2(12)$ & $5,1(6)$ & 0,238 \\
\hline Visitas regulares às escolas. & $6,8(8)$ & $5,1(6)$ & 0,791 \\
\hline $\begin{array}{l}\text { Acompanhamento das compras dos produtos alimentícios } \\
\text { - Licitação. }\end{array}$ & $8,5(10)$ & $7,6(9)$ & 1,000 \\
\hline Acompanhamento das compras da agricultura familiar. & $34,7(41)$ & $7,6(9)$ & $<0,001$ \\
\hline Visita técnica aos fornecedores. & $11,9(14)$ & $33,9(40)$ & 0,001 \\
\hline $\begin{array}{l}\text { Desenvolvimento de capacitação de Manipuladores de } \\
\text { Alimentos (Merendeiras). }\end{array}$ & $6,8(8)$ & $5,1(6)$ & 0,791 \\
\hline Interação e assessoramento (trabalho em equipe) ao CAE. & $13,6(16)$ & $8,5(10)$ & 0,327 \\
\hline \multicolumn{4}{|l|}{ Dimensão 3} \\
\hline Planejamento de cardápios, conforme a legislação vigente. & $9,3(11)$ & $3,4(4)$ & 0,118 \\
\hline $\begin{array}{l}\text { Aplicação do teste de aceitabilidade seguindo critérios da } \\
\text { legislação. }\end{array}$ & $35,6(42)$ & $29,7(35)$ & 0,494 \\
\hline Elaboração de Manual de Boas Práticas. & $16,9(20)$ & $44,9(53)$ & $<0,001$ \\
\hline $\begin{array}{l}\text { Verificação do perfil da população atendida, identificando } \\
\text { crianças com necessidades nutricionais especiais. }\end{array}$ & $22,0(26)$ & $24,6(29)$ & 0,788 \\
\hline Monitoramento do estado nutricional dos estudantes. & $21,2(25)$ & $23,7(28)$ & 0,784 \\
\hline
\end{tabular}

${ }^{*}$ Teste qui-quadrado de ajustamento

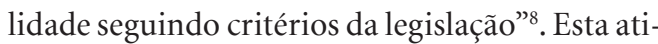
vidade é trabalhada em um momento específico do curso e, assim como era observado durante a formação, poucos participantes (11,9\%) "sempre desenvolveram", pois a metodologia para aplicar o teste ainda não havia sido validada para o uso na AE e somente foi padronizada pelo FNDE em 2009. Em 2007 e 2008 utilizou-se o material contido na metodologia de formação.

É de extrema importância atentar para o fato de que a formação e a informação sozinhas dificilmente poderão operar modificações na reali- 
dade. É preciso que mudem ou que sejam favoráveis também os contextos em que o profissional se insere. Ao encontro desse debate, Belik e $\mathrm{Chaim}^{16}$, ao fazerem uma análise de prefeituras que obtiveram destaque no Prêmio Gestor Eficiente da Merenda Escolar, chamam a atenção de que o desafio maior para o êxito do PNAE pode não ser o financeiro por si só, mas sim a construção de uma rede de apoio local (da área de educação e outros segmentos, gestores, sociedade civil) que, compartilhando ações, permita e sustente uma maior eficiência do Programa.

\section{Dificuldades encontradas pelos nutricionistas para implementar mudanças na realidade de trabalho}

Foi solicitado aos respondentes que indicassem quais as dificuldades encontradas na implementação dos conhecimentos construídos na formação. Dados esses importantes para aproximar os pesquisadores dos impasses da realidade que concorrem para uma menor expressividade da efetividade da formação. O impedimento mais referido pelos participantes foi a carga horária insuficiente para desenvolver inúmeras atribuições na $\mathrm{AE}$ no município. $\mathrm{O}$ que se verifica é que o nutricionista tem carga horária parcial junto à $\mathrm{AE}$ ou assume o programa paralelamente a atividades no setor saúde.

Quanto à dimensão 1, os respondentes revelaram uma grande dificuldade de adaptar todo este arcabouço técnico à realidade em que trabalham. O fator "estar cientes das atribuições ${ }^{17}$ dos nutricionistas" foi relatado como importante, embora seja de difícil aplicação.

Constatou-se que a maior parte das dificuldades se concentra na dimensão 2 (da gestão), e estão centradas na gestão de pessoas. Muitas falas ressaltam a resistência dos diversos atores do PNAE à mudança. Além disso, os nutricionistas declaram ter muitas dificuldades com os gestores, que não valorizam o Programa e não têm conhecimento sobre o trabalho do profissional de nutrição, gerando ausência de autonomia e falta de apoio da administração para o desenvolvimento das atividades. O relacionamento com os manipuladores é outro fator referido, gerando muita dificuldade de comunicação, e, principalmente, resistência às boas práticas sugeridas. A integração do Programa com a agricultura familiar está dentro das dificuldades de gestão mais relatadas. Finalmente, aparecem os processos licitatórios como sendo, ainda, muito complicados e de difícil apropriação, relatando a dificuldade de entrosamento com as equipes de compras municipais e estaduais.

Percebe-se que os entraves relacionados à gestão e à relação com manipuladores, gestores e outros atores é algo que está posto e, como destacado anteriormente, parece ser um dos grandes propulsores para o êxito do Programa. Neste sentido, e para compreender tal conjuntura, Domene $^{18}$ aponta para questões relacionadas à história do PNAE. O fato do Programa ter apresentando um caráter paternalista e assistencialista, e nem sempre ser tratado pelos próprios setores educacionais como peça importante para uma educação de qualidade, levou à construção, nas municipalidades, de um ambiente em que a alimentação é um apêndice ou adjuvante, como define a autora, o que parece interferir na interlocução do nutricionista com os demais gestores e pessoas ligadas à educação.

$\mathrm{Na}$ dimensão 3, as dificuldades foram muito específicas e se concentram na confecção do cardápio, cálculo do valor nutricional, elaboração das fichas técnicas, aplicação do teste de aceitabilidade e elaboração de manual de boas práticas. Isso demonstrar que até mesmo na execução técnica, o nutricionista se depara com a necessidade de se adaptar à realidade local, ousar e envolverse com outros atores, indo além do saber técnico, como destacam Ferreira e Magalhães ${ }^{19}$. Não basta saber, é preciso articular também o político e o ético com a responsabilidade e criatividade, para assim (re)fazer em cima das dificuldades ${ }^{20}$. Este é, sem dúvida, um cenário desafiador tanto para o desempenho da política e do nutricionista que a executa, quanto para aqueles que realizam ações de formação para esses atores do PNAE.

\section{Conclusão}

Buscando verificar a percepção dos nutricionistas quanto à formação, observou-se que no que diz respeito aos objetivos traçados a formação se revelou efetiva: na sua função de difusão e atualização das normas e objetivos do PNAE e na troca de experiências e práticas eficientes de gestão.

Esta metodologia trouxe algumas dificuldades em sua elaboração e execução, principalmente por não haver na literatura trabalhos que avaliem cursos de formação. Sendo assim, observase a necessidade de novos trabalhos nesta área.

Ainda, constatou-se as atividades e os assuntos que necessitam ser aprofundados, como aqueles que destacadamente ainda não são desenvolvidos pelos nutricionistas ou apresentam 
empecilhos para a execução. Com relação às dificuldades para a execução e aplicação de atividades junto ao PNAE, tal como abordadas na formação, verificou-se que as principais estão relacionadas à carga horária e à gestão de pessoas.

Este estudo aponta a necessidade de dar continuidade ao processo de formação, com cursos mais específicos de atualização e, ainda, cursos para outros atores que compartilham a experiência do PNAE com o nutricionista.

\section{Colaboradores}

ALS Scarparo e VR Oliveira participaram da estruturação do projeto, análise e interpretação dos dados, elaboração do artigo. JMV Bittencourt participou da estruturação do projeto, análise e interpretação dos dados. ENF Ruiz participou da proposta, estruturação do projeto e elaboração do artigo. PF Fernandes participou da estruturação do projeto. JZ Zys elaborou o banco de dados. CC Moulin participou da proposta do projeto.

\section{Referências}

1. Brasil. Portaria Interministerial no 1.010 de $08 \mathrm{de}$ maio de 2006. Institui as diretrizes para a promoção da alimentação saudável nas escolas de educação infantil, fundamental e nível médio das redes públicas e privadas, em âmbito nacional. Diário Oficial da União 2006; 9 maio.

2. Scarparo ALS, Moulin CC, Ruiz EF, Schuch I, Araújo JS, Souza M, Fernandes PF, Baldasso PL, Rocha PB, Bosa VL, Oliveira ABA. Ações do Centro Colaborador em Alimentação e Nutrição do Escolar da Universidade Federal do Rio Grande do Sul. Rev HCPA 2010; 30(3):302-305.

3. Brasil. Lei $n^{\circ} 11947$ de 16 de junho de 2009. Dispõe sobre o atendimento da alimentação escolar e do programa dinheiro direto na escola aos alunos da educação básica. Diário Oficial da União 2009; 17 jun.

4. Mota PR. Avaliação na administração pública: Eficiência, eficácia e efetividade. In: Caravantes GR, Bjur W, organizadores. Re-Administração em Ação. São Paulo: Makron Books; 1996.

5. Draibe SM. Avaliações de Implementação: esboço de uma metodologia de trabalho em políticas públicas. In: Barreira MCN, Carvalho MCB, organizadores. Tendências e Perspectivas na Avaliação de Políticas e Programas Sociais. São Paulo: IEE, PUC-SP; 2001.

6. Figueiredo AMC, Figueiredo MF. Avaliação política e avaliação de políticas: um quadro de referência teórica. Anál e Conj 1986; 1(3):107-127.

7. Centro Colaborador em Alimentação e Nutrição do Escolar da Universidade Federal de São Paulo. Manual de Capacitação de Nutricionistas. São Paulo: UNIFESP, CECANE - Campus Baixada Santista; 2007.

8. Brasil. Resolução no 38 de 16 de julho de 2009 . Dispõe sobre o atendimento da alimentação escolar e do Programa Dinheiro Direto na Escola aos alunos da educação básica. Diário Oficial da União 2009; 17 jul.

9. Arretche MTS. Uma Contribuição para fazermos Avaliações menos Ingênuas. In: Barreira MCN, Carvalho MCB, organizadores. Tendências e Perspectivas na Avaliação de Políticas e Programas Sociais. São Paulo: IEE, PUC-SP; 2001.
10. Encuestafacil: Convierte ahora la información en beneficio [Software de ferramenta web de pesquisas]. Madri: Encuesta Fácil; s.d.

11. Freitas H, Janissek R, Moscarola J. Dinâmica do processo de coleta e análise de dados via web. Apresentado no Congresso Internacional de Pesquisa Qualitativa; 2004 Mar 24-27; Taubaté/SP; 2004.

12. Babbie E. Métodos de Pesquisas de Survey. Belo Horizonte: Editora UFMG; 1999.

13. Voos AC. Atuação do nutricionista no Programa Nacional de Alimentação Escolar no estado do RS [monografia]. Porto Alegre: Universidade Federal do Rio Grande do Sul; 2009.

14. Ceccim RB. Educação Permanente em Saúde: desafio ambicioso e necessário. Interface Comun Saúde Educ 2005; 9(16):161-168.

15. Caniné ES, Ribeiro VMB. A prática do nutricionista em escolas municipais do Rio de Janeiro: um espaço-tempo educativo. Ciênc. educ 2007; 13(1):47-70.

16. Belik W, Chaim NA. O programa nacional de alimentação escolar e a gestão municipal: eficiência administrativa, controle social e desenvolvimento local. Rev. Nutr 2009; 22(5):595-607.

17. Conselho Federal de Nutricionistas. Resolução no 465 de 25 de agosto de 2010. Dispõe sobre as atribuições no Nutricionista, estabelece parâmetros numéricos mínimos de referência no âmbito do Programa de Alimentação Escolar e dá outras providências. Diário Oficial da União 2010; 26 ago.

18. Domene SMA. A escola como ambiente de promoção da saúde e educação nutricional. Psicol USP 2008; 19(4):505-517.

19. Ferreira VA, Magalhães R. Nutrição e promoção da saúde: perspectivas atuais. Cad Saude Publica 2007; 23(7):1674-1681.

20. L'Abatte S. Educação e serviços de saúde: avaliando a capacitação dos profissionais. Cad Saude Publica 1999; 15(Supl. 2):15-27.

Artigo apresentado em 09/10/2012

Aprovado em 17/11/2012

Versão final apresentada em 20/12/2012 